International Journal of Computer Science, Engineering and Information Technology (IJCSEIT), Vol.2, No.2, April 2012

\title{
THE REDUCTION IN COMPUTATION TIME IN THE COLOR IMAGE DECOMPOSITION WITH THE BIDIMENSIONAL EMPIRICAL MODE DECOMPOSITION APPROACH
}

\author{
Faten Ben Arfia ${ }^{1}$, Abdelouahed Sabri ${ }^{3}$, Mohamed Ben Messaoud ${ }^{2}$ and Mohamed \\ Abid $^{1}$ \\ ${ }^{1}$ Computer Engineering System design Laboratory (CES) \\ National Engineering School of Sfax: Tunisia \\ benarfia_faten@yahoo.fr \\ mohamed.abid@enis.rnu.tn \\ ${ }^{2}$ Laboratory of Advanced Technologies of medicine and signals (UR-ATMS) \\ National Engineering School of Sfax: Tunisia \\ M.benMessaoudeenis.rnu.tn \\ ${ }^{3}$ Laboratory of Electronics Signals, Systems and Computers \\ Faculty of Sciences, Dhar El mahraz FES. Morocco. \\ abdelouahed.sabri@gmail.com
}

\begin{abstract}
This paper presents two different approaches in color image decomposition domain with Bidimensional Empirical Mode Decomposition (BEMD). The first approach applies the BEMD on each channel separately and the second is based on interpolation of each channel in the sifting process. The comparison of two approaches shows the same performance of each approach in terms of visual quality, but they do not provide the same results in execution time which presents the most important criteron in real time applications. It was shown that the second BEMD approach based on interpolation of each channel in the sifting process, gives a gain in the point of view the execution time.
\end{abstract}

\section{KEYWORDS}

BEMD, IMF, Color image, interpolation, PSNR

\section{INTRODUCTION}

The field of digital image processing, especially the color images, has experienced continuous and significant expansion in recent years. So processing color images has received increased attention lately due to its importance in applications, such as multimedia technologies and telecommunications [1], where color image have an important role in the advertising and dissemination of information throughout the media.

Among the methods of image decomposition existing in the literature for example wavelet [2][3], Empirical Mode Decomposition (EMD) [4] is a flexible technique of signal decomposition. The basic idea is to decompose a signal into a sum of components, each of is a zero mean waveform, modulated in amplitude and frequency. It has a self-adaptive nature that enables it to identify changes in the signal, whether in terms of amplitude or frequency. Research proved that it is a 
very powerful tool for adaptive multi-scale analysis of non-stationary and nonlinear signals and took a place among the best known decomposition filters.

It is an extension to two-dimensional case, helped to develop new approaches for processing and analyzing multi-scale images and its use has led to better results than other decomposition techniques [5].

The BEMD (Bidimensional EMD) is particularly suitable for texture analysis. Huang et al. compare their results with those obtained by methods based on wavelet decomposition or Gabor Transforms, or diffusion [2] and show that the BEMD method is similar to nonlinear filtering depending of time and is self-adaptive.

Interpolation is an important issue both in the field of image signal for the estimation and the extraction of the IMF's from extremas. Different procedures can be used to calculate average envelope [4]. A natural extension of cubic splines for image is the thin-plate that is a special case of the basic interpolation functions for radial basis (RBF), however we could use other functions as a function of basis for the RBF.

In this paper, we propose an extension of the BEMD color images decomposition with two techniques: the first applies the BEMD on each channel separately, and recombines the three decomposition components of the image. The second technique is based on interpolation of each channel in the sifting process and the different IMFs are obtained after the summation interpolation images. The experimental part includes a comparative study of these two techniques at the base of computing time and the quality of the reconstructed image.

The rest of this paper is organized as follows: Section 2 gives a brief overview of the BEMD process. Section 3 describes the two modified approaches of color image decomposition. Then the paper continues with Section 4 which contains the experimental results and discussions. Finally, Section 5 concludes the paper.

\section{Bidimensional EMPIRICAL Mode Decomposition}

EMD is an adaptive decomposition of signals [6], introduced by Huang [4] for one dimensional data and then extended to Bidimensional signals [2]. The novel BEMD approach is either a highly adaptive decomposition [7]. It is based on the characterization of the image with this decomposition in Intrinsic Mode Function (IMF) where the image can be decomposed into a redundant set of composite images called IMF and a residue. Adding all the IMFs with the residue reconstructs the original image without distortion or loss of information. An IMF is characterized by two specific properties [8]:

- The number of zero crossing and the number of extremas points is equal or differs only by one.

- It has a zero local mean.

The algorithm is described as follows [9]:

1. Initialization: $\mathrm{r}_{0}=\mathrm{A}_{0}$ (the residual) and $\mathrm{k}=1$ (index number of IMF)

2. Extraction of the $k^{\text {th }} I M F: I_{k}(m, n)$

a. Initialization: $\mathrm{E}_{0}(\mathrm{~m}, \mathrm{n})=\mathrm{r}_{\mathrm{k}-1}(\mathrm{~m}, \mathrm{n})$ and $\mathrm{j}=1$

b. Extract the local extremas of $\mathrm{E}_{\mathrm{j}-1}(\mathrm{~m}, \mathrm{n})$ (minima and maximum) 
International Journal of Computer Science, Engineering and Information Technology (IJCSEIT), Vol.2, No.2, April 2012

c. Interpolate the local extremas to construct the upper and the lower envelope respectively $\operatorname{Envmin}_{j-1}(m, n)$ and $\operatorname{Envmax}_{j-1}(m, n)$

d.Calculate the average of the two envelopes:

$m_{j-1}(m, n)=\operatorname{Envmin}_{\mathrm{j}-1}(\mathrm{~m}, \mathrm{n})+\mathrm{E} v \max _{\mathrm{j}-1}(\mathrm{~m}, \mathrm{n}$

e. Update :

$E_{j}(m, n)=E_{j-1}(m, n)-m_{j-1}(m, n)$

f. Calculate the stopping criterion

$S D(j)=\frac{1}{N} \sum_{t=0}^{T}\left[\frac{\left(E_{j-1}(m, n)-E_{j}(m, n)\right)^{2}}{E^{2}{ }_{j-1}(m, n)+\xi}\right]$

Where $\xi$ is a term (low) to eliminate possible division by zero.

g.Decision: Repeat steps (b) to (f) until $\mathrm{SD}_{\mathrm{i}} \leq \mathrm{SD}_{\max }$, and then put

$$
\mathrm{I}_{\mathrm{k}}(\mathrm{m}, \mathrm{n})=\mathrm{E}_{\mathrm{j}}(\mathrm{m}, \mathrm{n})
$$

3. Update residual

$r_{k}(m, n)=r_{k-1}(m, n)-I_{k}(m, n)$

4. Repeat steps $1-3$ with $j=j+1$ until the number of extremas in $r j$ is less than 2 .

The sum of all modes, added to the residual component reconstructs the original image [8]:

$$
A(m, n)=\sum_{k=1}^{K} I_{k}(m, n)+r(m, n), k \in N^{*}
$$

\section{THE MODIFIED BEMD APPROACH TO COLOR IMAGE DECOMPOSITION}

Color images consist of three channels, such as red (R), green (G), and blue (B) channels. Thus, a color image can be viewed as a combination of three images channel [10].

If we consider a color image of size $(256 \times 256 \times 3)$, image contains 65,536 pixels in total, and since we have three values for each pixel, the decomposition of such image directly by BEMD will very costly in execution time.

The color image can be transformed into three channels R, G and B. So in this case, one applies the BEMD approach for each channel.

In this paper, we propose two possible approaches to BEMD color image decomposition: 
The first approach is to apply the BEMD on each channel separately. We obtained a set of IMF In each channel and with the summation of this IMFs and the residue, we can be found the reconstructed image for each channel. With the summation of three reconstructed image for each channel we obtained the result image. The process decomposition approach with the first approach of BEMD is illustrated in Figure 1.

In the second approach we apply the BEMD approach in each channel separately. But in the interpolation phase, the three interpolated images are combined in a single interpolated image (reconstructed according RGB channels of color images). Then the BEMD approach is continued until extract all the IMFs and the residue, and finally the reconstructed image. The process of the second BEMD of color image decomposition is illustrated in Figure 2.

Another way, in the second approach we collects the three channels just after the interpolation step. So until the interpolation the two approaches are the same, why we can say that both approaches are mathematically equivalents and as a result they give the same visual quality.

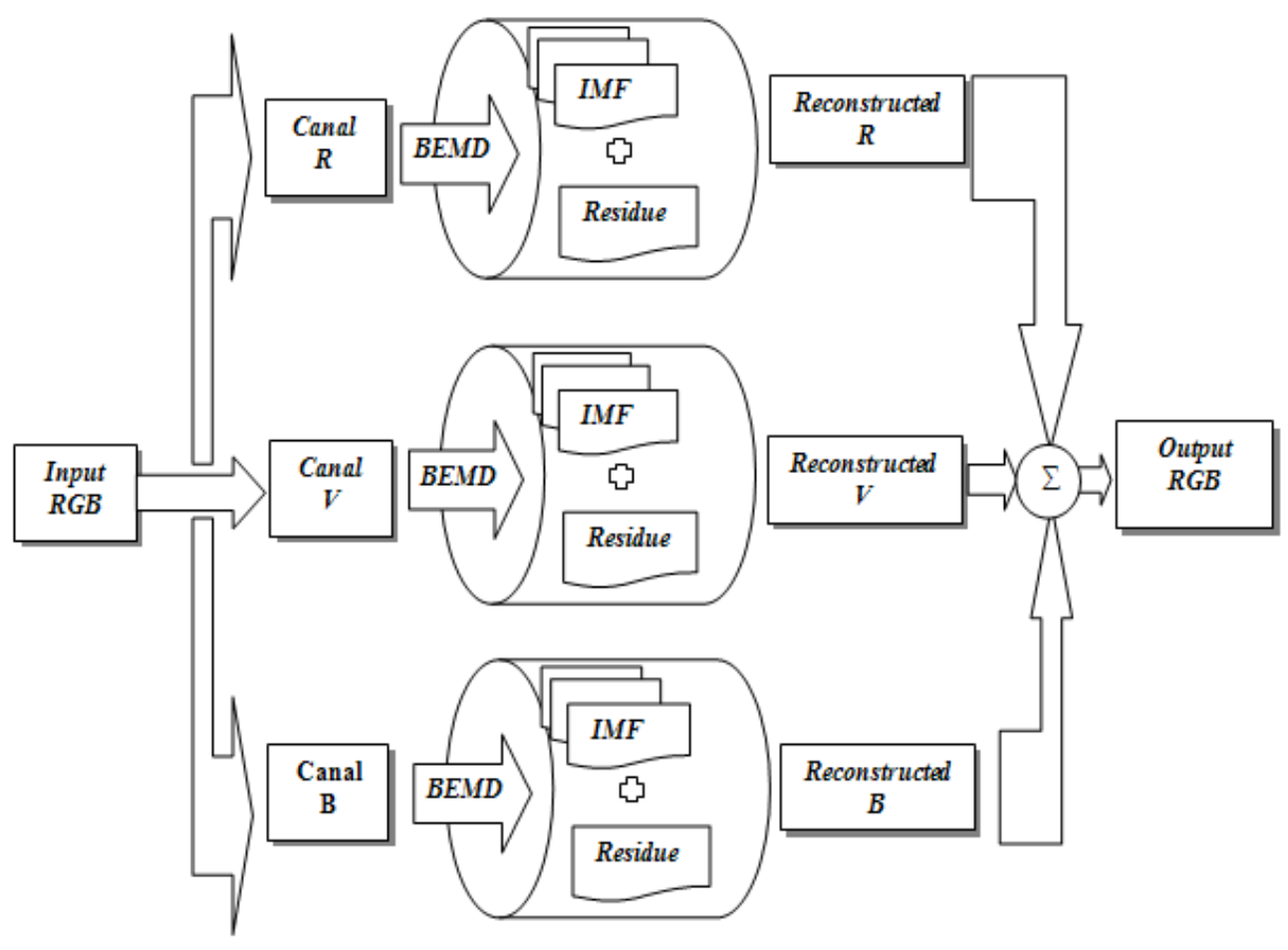

Figure 1. The first approach: The application of the BEMD on each channel separately for color image decomposition 


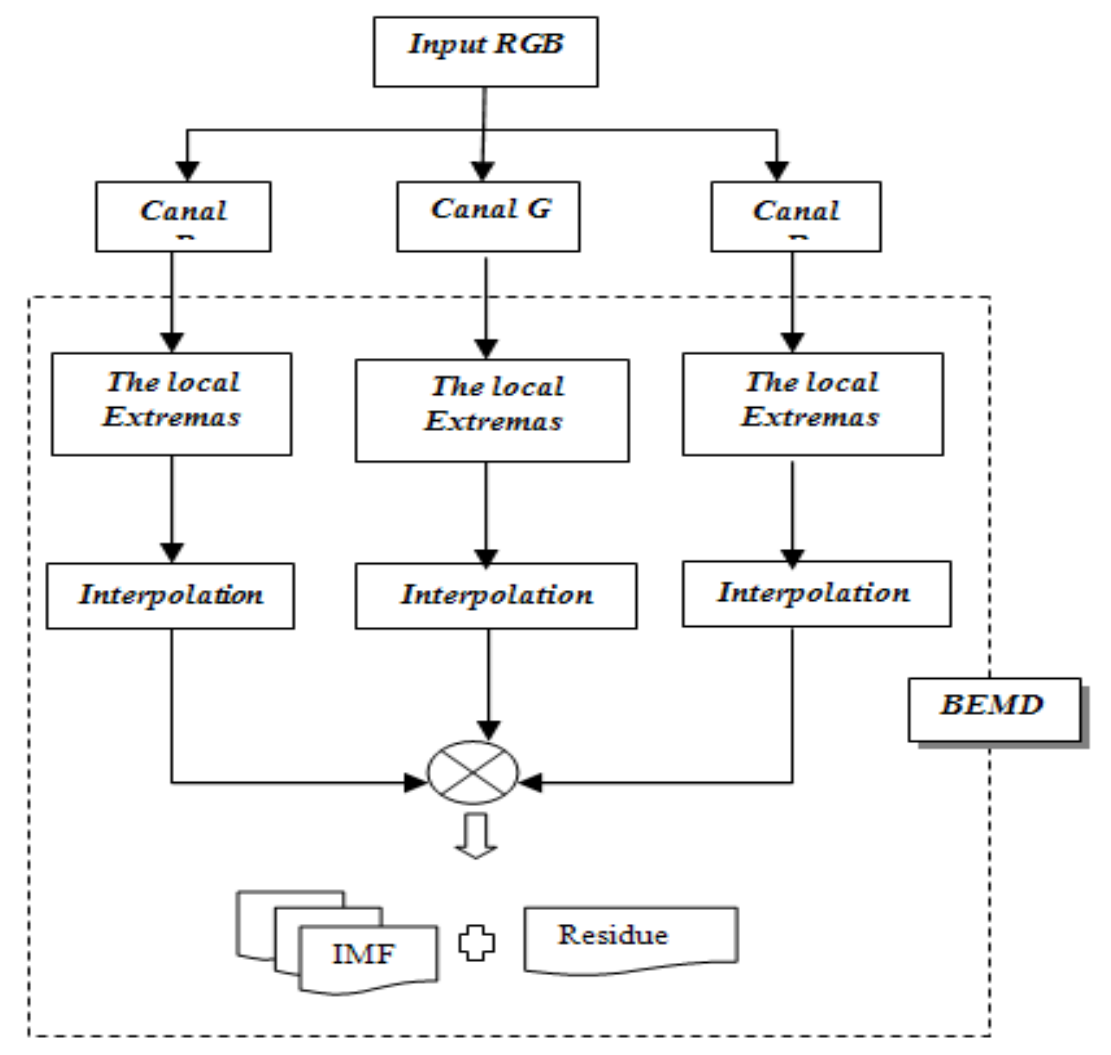

Figure. 2. The second approach: the application of the interpolation of each channel in the BEMD approach for color image decomposition

\section{EXPERIMENTATION AND RESULTS}

This section treats the implementation of two BEMD approaches to color image decomposition cited in the previous paragraph. The Figure 3 shows the results of two approaches of BEMD decomposition for color image of size $(256 \times 56 \times 3)$.

For each approaches, we have introduced the different IMFs with their residues. The last image (h) corresponds to the reconstructed image that permits a visual assessment of the BEMD efficiency.

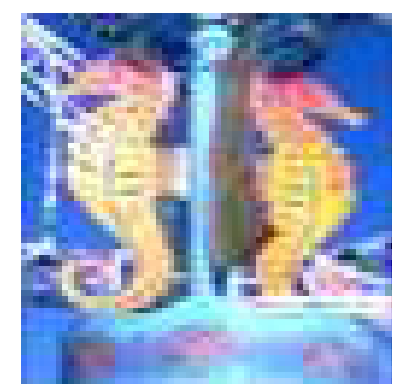

(A) Original RGB image 


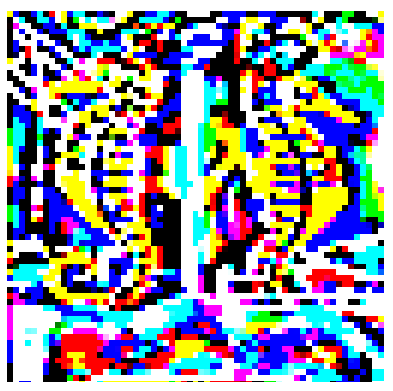

(B) First IMF

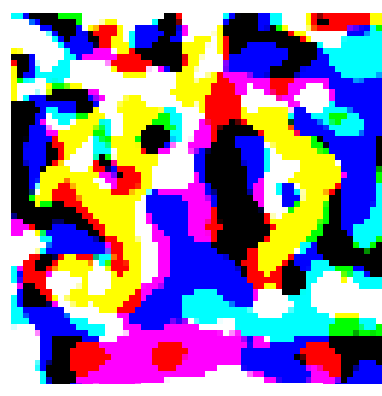

(D) Second IMF

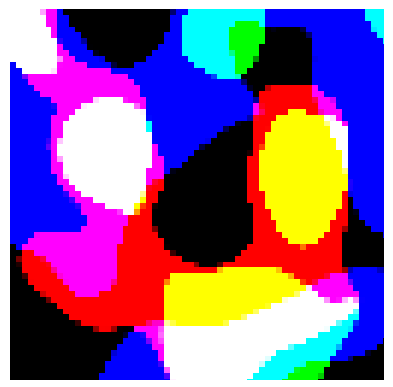

(F) Third IMF

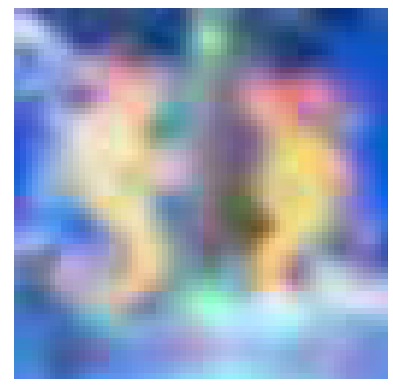

(C)Fist residue

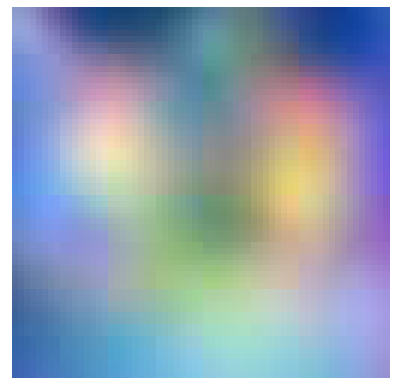

(E) Second residue

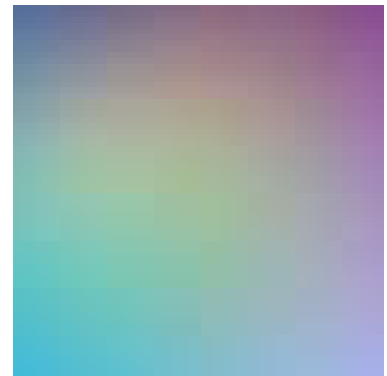

(G) Third residue

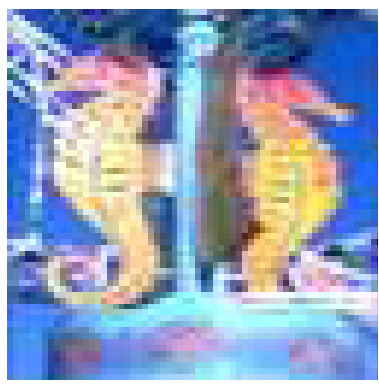

(H) Reconstructed RGB image

Figure. 3. Color image decomposition with BEMD (first and second) approach

The table 1 presents the number of the max and the min of each decomposition of IMF. 
Table 1. Number of extremas (min and max) of BEMD approach

\begin{tabular}{|c|c|c|}
\hline & max & min \\
\hline First IMF & 364 & 388 \\
\hline Second IMF & 40 & 72 \\
\hline Third IMF & 4 & 4 \\
\hline
\end{tabular}

First, we observe that as in the case of grayscale images, the decomposition led to the successful extraction of IMFs. Each IMF has oscillations in different frequency ranges from the highest frequencies for the first IMF to lower frequencies for residue with the minimal extrema for the latter. The simulation result shows that the second approach of the BEMD based in the interpolation in each canal is more effective then the first in term of visual quality and the a PSNR index(table 2).

Table 2. Comparison results for two approaches of color image decomposition with BEMD in term of PSNR $(\mathrm{dB})$

\begin{tabular}{|c|c|}
\hline The first approach & The second approach \\
\hline 45.7 & 46 \\
\hline
\end{tabular}

The Table 3 presents the different values of execution time for the BEMD methods of the color image of figure 3 .

Table 3. Execution time by the two proposed approaches of BEMD

\begin{tabular}{|c|c|c|c|}
\hline \multicolumn{3}{|c|}{ The first approach } & The second approach \\
\hline R & G & B & \\
\cline { 1 - 2 } $5.82(\mathrm{~s})$ & $5.78(\mathrm{~s})$ & $5.79(\mathrm{~s})$ & \multirow{2}{*}{$11.27(\mathrm{~s})$} \\
\hline \multicolumn{3}{|c|}{$17.4(\mathrm{~s})$} & \\
\hline
\end{tabular}

According to Table 3, which indicates the computational time of the two proposed approaches, the results show the interest of using the second approach if it is a large color pictures or an implementation in real time.

\section{Conclusion}

In this work, we presented two techniques for implementing the BEMD for color images decomposition. The BEMD applied for the RGB channels separately and the IMFs calculation from the summation of the interpolated image in each channel. Simulation results of decomposition images show that this second method is very interesting and promising for color images and can be applied in several areas that require the decomposition for the treatment of specific bands of frequencies in color images. In terms of comparison, the second technique is costly less in computation time. This technique seems better compared to the decomposition methods, and restoration of color images. 
International Journal of Computer Science, Engineering and Information Technology (IJCSEIT), Vol.2, No.2, April 2012

\section{REFERENCES}

[1] N. Papamarkos, C. Strouthopoulos and I. Andreadis, (2000) "Multithresholding of color and gray-level images through a neural network technique" Image and Vision Computing, pp.13-222

[2] S. G. Mallat, (1989) "A theory for multiresolution signal decomposition: the wavelet representation" IEEE Trans. Pattern Analysis and Machine Intelligence, Vol 11, No.7., pp. 674-693.

[3] L. Baisa Gunjal, N.M Suresh (2011) "Secured color image watermarking technique in DWT-DCT domain", International Journal of Computer Science, Engineering and Information Technology (IJCSEIT), Vol.1, No. 3.

[4] N. Huang, E., Z. Shen, SR. Long, MC. Wu, HH. Shih, Q. Zheng, N-C. Yen, CC. Tung and HH. Liu,1998 "The empirical mode decomposition and the Hilbert spectrum for non-linear and nonstationary time series analysis" Proc R Soc Land Ser A 454, pp. 903-995

[5] J. C. Nunes, Y. Bouaoune, E. Delechelle, O. Niang and Ph. Bunel , (2003) "Image analysis by Bidimensional Empirical Mode Decomposition” Image and Vision Computing 21 (12), pp.10191026

[6] A. Linderhed, (2009) "Image Empirical Mode Decomposition: a New Tool for Image Processing" Advances in Adaptive Data Analysis 1(2), pp. 265-294.

[7] F. Ben Arfia, M. Ben Messaoud and M. Abid, (2010) "A New Image denoising Technique Combining the Empirical Mode Decomposition with a Wavelet Transform Technique" 17th International Conference on Systems, Signals and Image Processing.

[8] A. Linderhed, (2002) "2D empirical mode decompositions in the spirit of image compression" Proc. of SPIE, Vol. 4738 2002, pp.1-8.

[9] A. Sabri, M. Karoud, H. Tairi and A. Aarab, (2008) "Fast Bidimensional Empirical Mode Decomposition Based on an Adaptive Block Partitioning" IJCSNS International Journal of Computer Science and Network Security, VOL.8, No.11.

[10] J-F. Aujol and S. H. Kang, (2006) "Color image decomposition and restoration" Journal of Visual Communication and Image Representation, 17(4), pp. 916-928 


\section{Author}

\section{Faten Ben Arfia}

Was born in Sfax, Tunisia, on July 15, 1980. She received a MASTER degree in New Technologies of Dedicated Computer Systems from the National School of Engineers of Sfax (ENIS) in Juin 2005. Now, she is preparing a Ph.D. at the Micro technology and System on Chip laboratory in the National School of Engineers of Sfax, Tunisia. Her main areas of interest are image processing and the non linear adaptive filter in image processing.

\section{Mohamed Ben Messouad}

He received the "Docteur Ingenieur" degree in automatic control engineering from the University of Paul Sabatier of Toulouse, France, in 1983. Since 1983 and the Habilitation Degree in 2008, he is with the Department of Electric engineering from the University of Sfax, Tunisia as an Professor. He is also a Member of the Electronic and Information Technology Laboratory for Research on Information theory and Adaptive Control Systems. His current research interests include applied techniques in cardiology, artificial neural network, adaptive observers and their applications.

\section{Mohamed Abid}

Is a professor of electrical engineering at Sfax University in Tunisia He holds a Diploma in electrical engineering in 1986 from the University of Sfax in Tunisia and received his $\mathrm{PhD}$ degree in Computer Science in 1989 at the University of Toulouse in France. His current research interests include HardwareSoftware codesign, design space exploration and prototyping strategies for real-time system

\section{Abdelouahed Sabri}

Was Born in Azrou, Morocco, on March 25, 1978. Professor at the ENSAT Marocco. He received his Ph.D. in 2009 from the University Sidi Mohamed Ben Abdellah, Morocco. These research areas include systems analysis, image processing and information processing. 